\title{
Bio-compatibility of metallic alloys for body-area communication systems
}

\author{
Ildiko Peter ${ }^{1}$ \\ Department of Applied Science and \\ Technology \\ Politecnico di Torino- Italy \\ +39011090 4670 \\ ildiko.peter@polito.it
}

\author{
Mario Rosso ${ }^{2}$ \\ Department of Applied Science and \\ Technology \\ Politecnico di Torino- Italy \\ +390110904664 \\ mario.rosso@polito.it
}

\begin{abstract}
Mechanical behavior, biocompatibility in body environment and tissues and chemical stability are the most important requirements for the effective application of any bio-implant materials in the human body. Among the known metals and alloys, stainless steels, $\mathrm{CoCr}$ alloys and $\mathrm{Ti}$ and its alloys are the most widely used materials in such applications. In case of their use, one of the most important engineering approach is to guarantee insignificant biological stress to the human system and to maintain the whole integrity and functionality of the human being.

In this paper we pay our attention on the study of the properties of two types of metallic alloys: one of them is a modified $\mathrm{CoCr}$ alloy, while the second one is a new generation of TiNb based alloy. The first step of the study is related to the determination of the microstructural and mechanical behavior of the alloys and to assess their chemical stability by the evaluation of their corrosion resistance. The further step will be to evaluate and to control the interaction between the human body and the biomaterial inside the biological environment using an appropriate numerical model. In this way it will be possible to detect the presence of defects in the biomaterial, considering the surface chemistry and its topography, which can control this system leading to regulate in time an inappropriate interface avoiding the removal of the implant for the restoration. Furthermore, the results of such an investigation represent a good starting point in designing of intra-, inter-body or body-coupled printed implanted antennas, i.e., antennas located inside the body used to communicate with external base stations or between different nodes of a network.
\end{abstract}

\section{Categories and Subject Descriptors}

J. [Computer applications], J.3[Life and medical sciences]: Health, medical information system

K [Computing Milieux], K.4. [Computers and Society], K.4.2 [Social Issues]: - Handicapped persons/special needs

\section{General Terms}

Theory, Experimental results.

\section{Keywords}

Metallic biomaterials, $\mathrm{CoCr}$ alloys, Ti alloys, body environment, numerical simulation.

\section{INTRODUCTION}

Because of their excellent mechanical properties, good corrosion resistance and good biocompatibility, metallic alloys are usually employed for dental implants and orthopedic device production. In addition, they are desirable for some neuromuscular stimulation tools production, due to their good thermal and electric conductivity coming from the natural electron movement within the metal crystal system. Compared to polymeric, ceramic and composite based biomaterials, metals and their alloys have superior tensile and fatigue strength associated to a superior fracture toughness, good corrosion resistance and good biocompatibility [1, 2]. Usually, metallic alloys are exploited thanks to their structural functions in different parts of the human body to substitute the lost or unhealthy natural assembly and to repair them, but they do not have any bio-functionalities, like blood compatibility. The most important metallic biomaterials are stainless steel, $\mathrm{CoCr}$ based alloys and $\mathrm{Ti}$ and its alloys. In the case of stainless steel one can have localized corrosion and the release of metallic ions which could be dangerous for the human body. Actually, CoCr based alloys have acquired higher attention and compared to stainless steel as strength and corrosion resistance regards show superior features, but as from manufacturing point of view they are more expensive [3, 4]. For such alloys, $\mathrm{Cr}$ is a crucial constituent from corrosion resistance point of view and gives to the alloy intrinsic corrosion resistance, developing a passive chromium oxide layer. $\mathrm{Cr}$ and Mo act as solid solution strengthening elements and can modify the alloys thermal coefficient of expansion. $\mathrm{Ni}$ can amplifies the ductile properties of the $\mathrm{Cr}$-Co metallic alloy and can stabilize the faced centred cubic structure which is favored due to its better tenacity of the Co-matrix, but at the same time, $\mathrm{Ni}$ is a potent allergen component and can cause higher hypersensitivity reaction compared to other metallic or ceramic materials used in such application. Inflammatory responses, associated to $\mathrm{Ni}-\mathrm{Cr}$ alloy restoration subside when the alloys is removed and replaced with Ni-free materials [5, 6]. Actually, Ti and its alloys have been received much more attention and they show the best 
biocompatibility between the metallic biomaterials. They reveal good fatigue resistance, excellent in vivo corrosion resistance due to the stable and rapidly forms passive oxide layer. In addition, the lower elastic moduli compared to other metallic biomaterial, guarantee less stress shielding avoiding bone atrophy and faster osseointegration. The development of close bone-to-implant apposition after short implantation periods result an important benefit for permanent bone-interfacing implants [7]. A standard $\mathrm{Ti}$ based alloy for orthopaedic implants is Ti6Al4V alloy, which has been firstly developed for aeronautic applications. Another alloy, widely used as bio-alloy is NiTi alloy, thanks to its unique superelastic and shape memory properties. However, a lack of biocompatibility has been demonstrated with these alloys: $\mathrm{Ni}$ is very allergenic and can cause violent inflammatory reactions; in Ti6Al4V, Al ions released from implant are associated with long term health problems like neurological pathologies while $\mathrm{V}$ is also known to be cytotoxic. Moreover, the high Young's modulus of the Ti6Al4V alloy affects long-term practical performances due to the stress shielding effect. In the last period, new Ti alloys with $\alpha+\beta$ structure, without $\mathrm{V}$ were obtained: Ti6Al7Nb; Ti5Al2.5Fe; Ti6Al6Nb1Ta; Ti5Al3Mo4Zr. Since the $\beta$ microstructure in Ti alloys shows a significantly lower modulus than the $\alpha+\beta$ one [8], there is a need for improvement in design for an ideal medical implant. Consequently, a new generation of low modulus $\beta$-Ti alloys, free of $\mathrm{Ni}, \mathrm{V}$ an $\mathrm{Al}$ have been made with $\beta$-stabilizing and biocompatible elements such as $\mathrm{Nb}, \mathrm{Ta}, \mathrm{Zr}$ and $\mathrm{Mo}$ (alloys of super $\beta$-type - Ti13Nb13Zr and $\beta$-type Ti15Mo). Among the $\beta$-type Ti alloys, the biocompatible metastable $\beta \mathrm{Ti}-\mathrm{Nb}$ based alloys are very promising for biomedical applications. Indeed, these alloys have a low elastic modulus close to that of bone. On the other hand, an encouraging super-elastic behavior can be achieved due to the amartensitic transformation (orthorhombic phase). Consequently, Ni-free alloys can also be designed for biomedical smart devices or orthodontic wires $[9,10]$. A group of special $\beta$-Ti alloys with unique physical-mechanical properties and wide range of possibilities in medical applications are Gum alloys, belonging to the $\beta$-type Ti alloys and is basically expressed as $\mathrm{Ti}(\mathrm{Ta}, \mathrm{Nb}, \mathrm{V})+$ ( $\mathrm{Zr}, \mathrm{Hf}, \mathrm{O})$. Gum alloys reveal excellent mechanical behaviour at room temperature: an ultra-low Young's modulus (60-70 GPa) and a non-linear elastic behaviour, an extended elastic limit, ultrahigh strength (> $1 \mathrm{GPa}$ ), superplastic-like deformability, Invar-like thermal expansion, and Elinvar-like thermal dependence of the elastic modulus. These properties provide numerous opportunities for Gum alloys application [11]. After their development in Japan, being the first alloy that combines extremely low elastic modulus with extremely high strength they became ideal candidate for medical implants able to withstand reasonably large loads, due to their special mechanical properties and with no hazardous elements in their chemical composition. $\mathrm{Nb}$, $\mathrm{Ta}$ and $\mathrm{Zr}$ are now considered to be the safest, non-toxic and non-allergic alloying elements for $\mathrm{Ti}$ alloys, being demonstrated through research studies their higher cell viability, corrosion resistance, tissue compatibility and nonallergic properties. One of the alloys studied in the present paper is situated in the compositional system: Ti-Nb-Ta-Zr-O, and the other one is a CoCr-based alloy. Metallic implants made of biodegradable metals or their alloys, like $\mathrm{Mg}$ and its alloys, reveal some new biomedical features: after being implanted, they will gradually degrade, avoiding their elimination associated to an immediate reduction in health risks, costs and damaging [12]. $\mathrm{Mg}$ and its biodegradable alloys (AZ91, WE43, AM50, LAE442) appear to be the most promising candidate for the construction of temporary implants, including stents [13], since they can degrade naturally in the physiological environment by corrosion and the corrosion products of such implants have been well accepted by human organism: $\mathrm{Mg} 2+$ ions are the fourth most abundant cation in the human body and are stored mainly in bones. In this general framework, the study and the development of new alloy compositions and new production processes for medical device construction can be integrated by numerical investigation on the development of efficient production processes and/or on the model of the possible interaction between the biomaterial surfaces and the body environment. Such studies allow drastically reduction of the number of experimental tests and the probability of infections. Theoretically modelling, in many cases, one can avoid the removal of the implant, the cure of the diseases, which is very important for the patient and is beneficial from economical point of view.

The aim of the present paper is to provide, firstly, a general overview on the metallic biomaterials and their possible use in the human body focusing the attention on Ti enriched CoCr-based and TiNb-based alloys. In the further step we propose a possible model to evaluate and to control the interaction between the metallic biomaterial and the surrounding biological environment using an appropriate numerical model. In this way we will control the surface chemistry and its topography, which can control this system leading to correct in time the inappropriate interaction between the two elements avoiding the removal of the implant for the restoration. Additionally, the development of the radiating part, i.e., antenna of an intra-, inter-body or body-coupled system could directly benefit from the possibility to have a biocompatible conductor with good electric properties.

\section{EXPERIMENTAL DETAILS}

CoCr basic alloy enhanced by $\mathrm{Ti}(25.5 \% \mathrm{Cr}, 6 \% \mathrm{Mo}, 1 \% \mathrm{Si}, 4 \% \mathrm{Ti}$, Co balance and the TiNb based alloy (Ti61.70, 30.50Nb, 4.80Zr, $2.80 \mathrm{Ta}, 0.20 \mathrm{O}$ ) were produced by cold crucible levitation melting technique with induction heating system.. As reference material a commercially employed Wirobond ${ }^{2} 280$ alloy (Co60.9, Cr25, Mo4.8, W6.2, Ga2.9) were used. The samples in as-cast state were prepared by traditional metallographic procedures and then were submitted to morphological and compositional analysis by Optical Microscope (OM, MeF4 Reichart-Jung) and by Scanning Electron Microscopy (SEM, Leo 1450VP) equipped with Energy-Dispersive $\mathrm{X}$-ray Spectrometry (EDS, Oxford microprobe). X-ray technique (X-ray, PANanalytical tool with $\mathrm{Cu} \mathrm{k} \alpha$ wavelength of $1.5418 \AA$ ) was employed for phase identification. Mechanical properties were evaluated by hardness measurement performed on the polished samples using a force of $5 \mathrm{~N}$ for $15 \mathrm{~s}$ for each measurement. The corrosion behaviour of the alloys was evaluted by static immersion test according to the route specified in the Standard ISO $10271 / 2011$ at $37^{\circ} \mathrm{C}\left( \pm 1^{\circ} \mathrm{C}\right)$. The samples were maintained in acid solution (7.5 ml lactic acid, $5.85 \mathrm{~g} \mathrm{NaCl}, 300 \mathrm{ml} \mathrm{H}_{2} \mathrm{O}$ di grade 2 purity, and $700 \mathrm{ml} \mathrm{H}_{2} \mathrm{O}$ ) at $\mathrm{pH}=7.4$, simulating the oral cavity surroundings. The samples were monitored after the permanence in this biological solution for 28 days. As reference solution a biological solution maintained in the same condition with no any metallic alloy inside was used. 


\section{EXPERIMENTAL EVALUATION AND CHARACTERIZATION OF THE METALLIC BIOMATERIAL}

The microstructural investigation, mechanical and corrosion resistance evaluation of the biomaterials considered for medical device production is directly correlated to the surface chemistry of the alloys and to their topography. The adhesion mechanism in the human body structure will be governed by these elements. For these reasons, in this paper we, firstly, experimentally characterize the metallic alloys and then we propose a theoretical study for the further evaluation of the interface created in the body between the metallic alloys and the adjacent atmosphere.

\subsection{Morphological observation}

The microstructure of the $\mathrm{CoCr}$ based alloy shows a nonhomogeneous features, and according to the ternary equilibrium diagram, the presence of the solid solution (dark areas in the Figs.1) is shown with a casual disposition of the dendrites within the structure (light areas in the Figs. 1); in addition carbides (mostly $\mathrm{Cr}_{23} \mathrm{C}_{3}, \mathrm{Cr}_{7} \mathrm{C}_{3}$ and $\mathrm{TiC}_{8}$ ) formation was identified. There is no evidence of continuous grain boundary carbides, which would be undesirable because negatively affect the ductility of the alloys. $\mathrm{Ti}$, due to its high affinity to the carbon, easily reacts with the carbon inside the metal matrix and the residual unbound element is dissolved in the solid solution. In the case of the commercial alloy the growth of the dendrites occurs according to a particular direction as revealed in Figure 1a and the structure results more uniform compared to the experimentally produced and $\mathrm{Ti}$ enriched alloy (Figure 1b) where the segregation tendency is more accentuated and the dendrites appear larger with indefinite orientation. A typical pure equiaxed $\beta$ grain microstructure for the $\mathrm{TiNb}$ alloy is reported in Figure 1c. The average $\beta$ grain size is about $161 \mu \mathrm{m}$.

\subsection{Mechanical behavior evaluation}

The carbide development confers to the alloy high hardness improving the alloy wear and corrosion resistances. Hardness measurements have been performed with a standard Vickers pyramidal Figure 2 reports the obtained results. As expected, the Ti modified alloy shows the higher hardness due to the highest content of $\mathrm{Cr}$ and Mo carbides, compared to the reference metallic alloy with no any carbide formation. As concerns, TiNb based alloy there are no any presence of hard particles and the hardness values are lower than those obtained for the commercially used metallic alloy (there are only the presence of $\beta$-Ti in the composition) and confers to the alloy good workability.
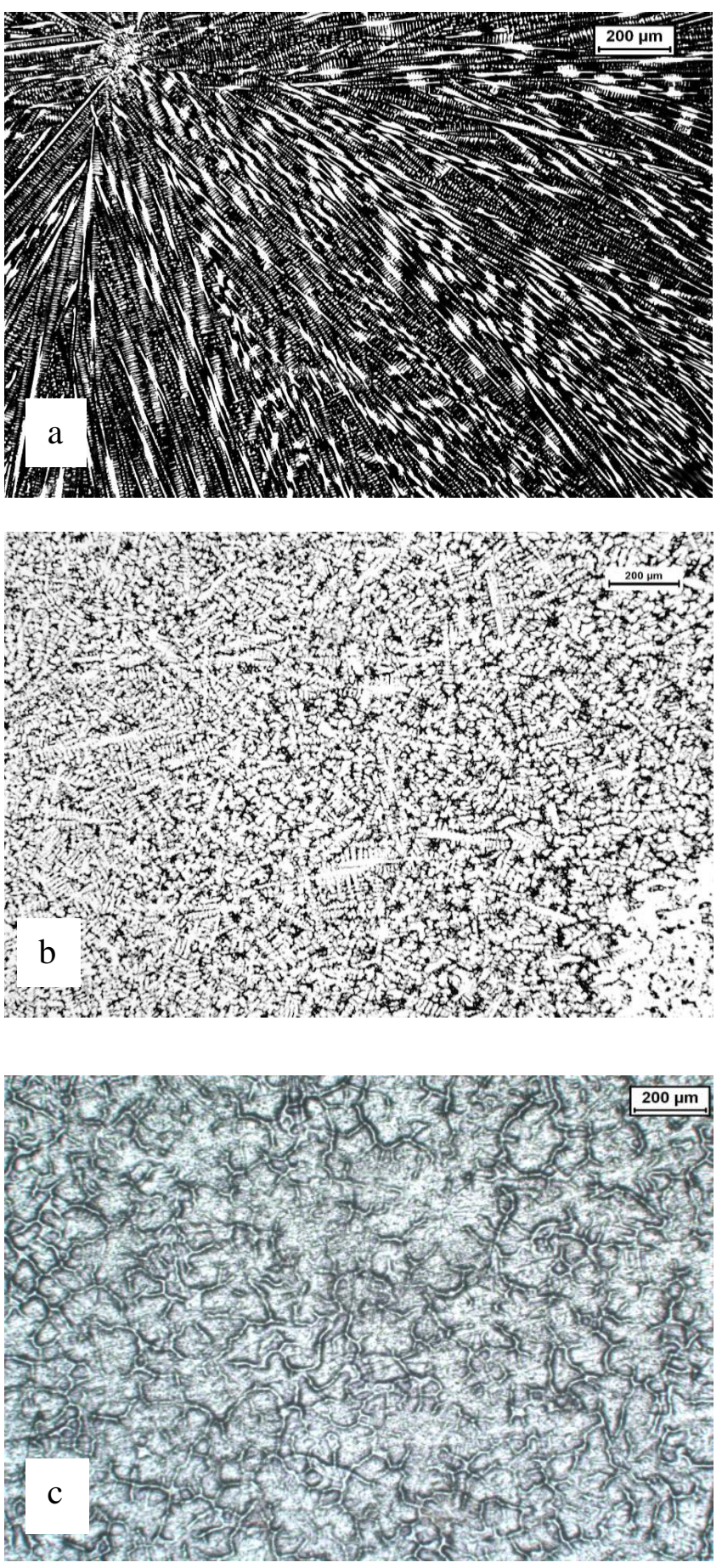

Figure 1: Optical micrographs of the alloys employed for the study: (a) Wirobond ${ }^{2} 280$ alloy, (b) $\mathrm{Ti}$ enriched $\mathrm{CoCr}$ alloy and (c) TiNb alloy 


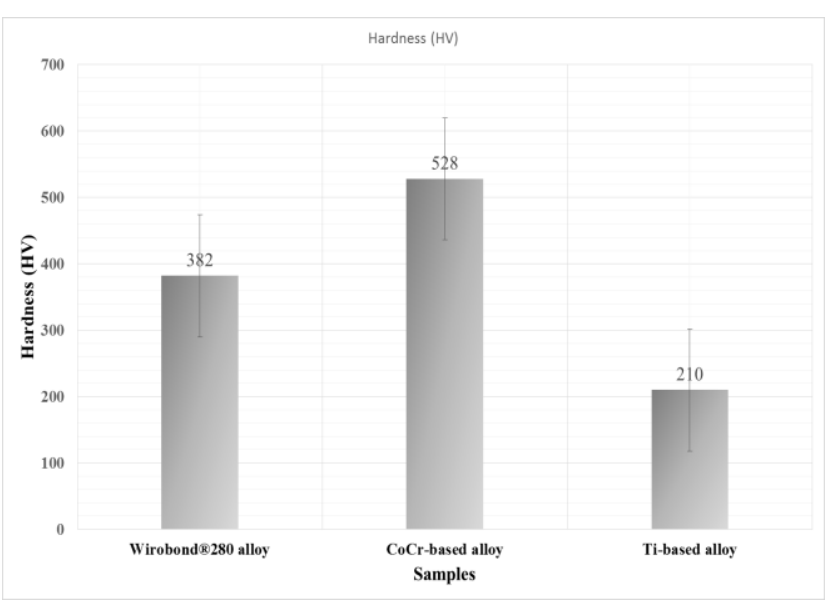

Figure 2: Vickers hardness measurement results for the alloys investigated

\subsection{Corrosion resistance evaluation}

As corrosion resistance regards, the samples have been surface-finished and then ultrasonically cleaned to remove any impurities and to avoid any possible contamination. The bottles used for the test have been disinfected. Each samples have been placed separately in the bottle and one bottle has been monitored containing only the solution. After a regular time, the weights of the samples have been measured and the $\mathrm{pH}$ values has been monitored. The alloys do not present any significant weight alteration after 28 days. During one week all tested materials maintain the initial $\mathrm{pH}$ value. No significant release of metallic ions has been detected even after 28 days, as reported in Figure 3. The result obtained has pointed out that the alloys conserve the condition requested: according to the Standard used for the test the deviation for the initial state could be only $1 \%$ and the experimentally widely fulfil this condition. The dissolution of the passive film developed on the surface of the alloy, with protective properties, has a very low rate and it is able to protect the alloy in such conditions, which simulates the oral cavity environment.

The morphological investigation pointed out that both experimentally metallic alloys are homogeneous as their structure concerns. They do not present on their surfaces inclusions, local depressions, in terms of dimples and cavities, which can favour the adhesion of the particles deriving from the biological environment. As their mechanical behaviour concerns, they are adequately resistant to withstand the mechanical solicitations and they do not present such a hardness that compromise their workability. It was observed that the alloys are corrosion resistant in a simulated human environment, since no important release of metallic ions has been detected for the period of 28 days. Based on the results obtained, the alloys show an adequate properties for biomedical purpose and the further step is to theoretically evaluate the possible parameters which can influence and can create the interaction between the biomaterial surfaces and the human body environment.

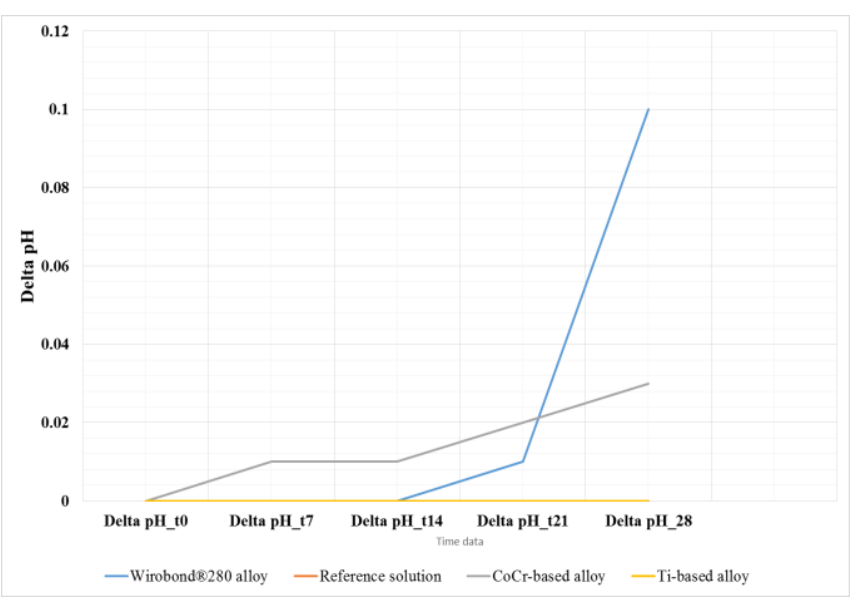

Figure 3: Variation of the $\mathrm{pH}$ value during 28 days of investigation

\subsection{Analytical model description}

In the case of the use of medical devices results very important to consider and to control the adhesion of different elements to the biomaterial surfaces. If adhesion occurs, this lead to the formation of a contagious layer on the medical devices and can conduct to the failure of the implant and diseases of the person. The adhesion is governed by different physical and chemical properties and is depends on the elements involved in the system: the substrate biomaterial and the biological environment. The hydrophobicity, hydrophilicity and the surface charge are the most important aspects in this context $[14,15]$. The interaction energies governing the initial phase of the adhesion mechanism are mostly related to the attractive forces of Van der Waals (LW) type and the electrostatic double layer (EL) interactions, which can be either attractive or repulsive depending on the surface charge. These interaction energies can be generally described by the classical Derjaguin, Landau, Verwey, Overbeek theory (DLVO) [16], and can be used to evaluate the adhesion energy variations involved in the process as a function of the separation distance between the interacting surfaces. By comparing the different adhesion energy variation and by comparing them one can predict in order to compare predict if the adhesion is favorable or not for the elements combination and which elements has a positive or negative effect on the adhesion mechanism.

The influence of the chemical composition and of the surface topography on the adhesion of the various particles coming from the biological environment will be determined using the DLVO theory. The DLVO theory assumes that the interaction forces can be well approximated by the total energy of adhesion due to the interpenetration of the electrical double layer. The force $F(h)$ among two units can be obtained using (1), considering a surface separation (h) and the free energy of the two units $W(h)$ per unit area:

$$
\mathrm{F}(\mathrm{h})=2 \pi R_{e f f} W(h)
$$




$$
R_{\text {eff }}=\frac{R_{+} R_{-}}{R_{+}+R_{-}}
$$

Where $\mathrm{R}+$ and $\mathrm{R}$ - are the radii of the two units involved in the considered process respectively.

Identifying the force between the materials, the interaction free energy is given by (3):

$$
U(h)=\int_{h}^{\infty} F\left(h^{\prime}\right) d h^{\prime}
$$

DLVO theory assumes that the free energy per unit area can be well approximated by two additive contributions: Van der Waals and double layer interactions (4) :

$$
W(h)=W_{\mathrm{vdW}}(h)+W_{\mathrm{dl}}(h)
$$

Van der Waals forces are almost always present, and they result from the interactions of the rotating or fluctuating dipoles of the atoms and the molecules. This type of interaction can be modeled by (5)

$$
W_{\mathrm{vdW}}(h)=-\frac{H}{12 \pi h^{2}}
$$

where $\mathrm{H}$ is a constant and in most situations is positive. Typical values of $\mathrm{H}$ are $10^{-21}-10^{-19} \mathrm{~J}$. A slight decrease of $\mathrm{H}$ occur when the salt level of the solution increases. The model takes into consideration also the flatness of the substrate material (the surface roughness can reduce this interaction at smaller distances), important aspect for the considered application. In case of low salt level and for charged substrates the double layer interactions are important. The interaction free energy can be approximated by (6):

$$
W_{\mathrm{dl}}(h)=\frac{2 \sigma_{+} \sigma_{-}}{\epsilon_{0} \epsilon \kappa} \exp (-\kappa h)
$$

where $\sigma^{+}$and $\sigma-$ are the surface charge densities per unit area of the two surfaces, $\varepsilon 0$ is the permittivity of vacuum, $\varepsilon$ the dielectric constant of water, and $\kappa$ is the inverse Debye length. The last parameter is given by (7):

$$
\kappa^{-1}=\left(\frac{k_{\mathrm{B}} T \epsilon_{0} \epsilon}{2 q^{2} N_{\mathrm{A}} l}\right)^{1 / 2}=\frac{0.3 \mathrm{~nm}}{\sqrt{l}}
$$

where $\mathrm{q}$ is the elementary charge, NA is the Avogadro's number, I is the ionic strength, $\mathrm{kB}$ is the Boltzmann constant, and $\mathrm{T}$ is the absolute temperature. The second equality is approximatively valid for water at room temperature.

\section{Conclusions and future directions}

In this paper, we investigated two metallic alloys for biomedical applications in the human body. The morphological analysis evidenced that both metallic alloys are homogeneous with no any presence of inclusions or local depressions. In addition, they are sufficiently strong to survive the mechanical solicitations. From corrosion resistance point of view, the considered alloys fulfil the conditions required for the release of metal ions in the simulated biological solution. As future work, we will consider the experimentally achieved results and we will use them to theoretically investigate on the parameters of the surface features which control the adhesion mechanism between the metallic substrate and body environment. As any communication system, e.g., intra-, inter-body or body-coupled, requires reduced size antennas, e.g., printed, for communication between sensors/devices inside the body and external base station. The studied metals exhibit good electric properties as conductibility, etc. making them a good candidate for considering them as ground plane or radiative elements for example when flat printed antennas are employed. Using this approach, it would be possible to monitor the human vital functions by placing such devices in the different helpful positions on and near the human body with the possibility to communicate with each other with no any negative health effects for the person. The results of the study demonstrates that the using such alloys without any further coatings is possible. Such solution allows reduction of the cost and of the dimensions of the devices.

\section{Acknowledgement}

This research has been carried out within the European ManuNet Framework Programme "Load-bearing implants functionalized of superelastic alloys"- acronym ISA".

\section{REFERENCES}

[1] Ashby MF and Jones DR. Engineering Materials 1. An Introduction to their Properties and Applications, and 2. An Introduction to Microstructures, Processing and Design, Pergamon Press: London, 1980.

[2] R.Z. Valiev, R.K. Islamgaliev, and I.V. Alexandrov, Bulk Nanostructured Materials from Severe Plastic Deformation, Prog. Mater. Sci., 45 (2000) 103.

[3] Kurtz, S.M., Ong, K., Bozic, K.J., Greenwald, A.S., Prevalence of primary metal-on-metal bearings in the United State. In: AAOS 2011 Annual Meeting, American Academy of Orthopedic Surgeons, San Diego, CA, USA. 2011.

[4] Thomann, J. Uggowitzer, Wear- corrosion behaviour of biocompatible austhenitic stainless steel, Wear 239 (2000) 48-58.

[5] M. K. Lei, X.N. Zhu, In vitro corrosion resistance of plasma source ion nitrided austenitic stainless steels Biomaterials 2 641-647 2001.

[6] M. Browne, P.J. Gregson, Surface modification of titanium alloy implants, Biomaterials 15 894-898 1994. [41] Collings EW.,The physical metallurgy of titanium alloys, Ohio: ASM; 1984. pp. 321-354.

[7] Polmear IJ. Light alloys. In: Metallurgy of the light metals.Metallurgy and materials science series. London: Arnold; 1981. 239-278.

[8] M. Niinomi, Shape memory, Super Elastic and Low Young's Modulus Alloys, Biomaterials for Spinal Surgery, edited by Luigi Ambrosio and Elzabeth Tanner, Woodhead Publishing, 2012, ISBN: 9781845699864.

[9] S. Miyazaki, H.Y. Kim, H. Hosoda, "Development and characterization of Ni-free Ti-base shape memory and 
superelastic alloys" Materials Science and Engineering: A, Volumes 438-440, 25 November 2006, Pages 18-24, doi:10.1016/j.msea.2006.02.054.

[10] Mitsuo Niinomi, "Recent research and development in titanium alloys for biomedical applications and healthcare goods", Science and Technology of Advanced Materials Volume 4, Issue 5, September 2003, Pages 445-454, doi:10.1016/j.stam.2003.09.002.

[11] Furuta, T., Kuramoto, S., Hwang, J., Nishino, K., \& Saito, T. (2005). Elastic Deformation Behavior of Multi-Functional Ti$\mathrm{Nb}-\mathrm{Ta}-\mathrm{Zr}-\mathrm{O}$ Alloys. MATERIALS TRANSACTIONS, 46(12), 3001-3007. doi:10.2320/matertrans.46.3001.

[12] Li J, Song Y, Zhang S, Zhao C, Zhang F, Zhang X, et al., In vitro responses of human bone marrow stromal cells to a fluoridated hydroxyapatite coated biodegradable $\mathrm{Mg}-\mathrm{Zn}$ alloy, Biomaterials 201031 5782-8.

[13] Kraus T, Fischerauer SF, Hanzi AC, Uggowitzer PJ, Loffler JF, Weinberg AM., Magnesium alloys for temporary implants in osteosynthesis: in vivo studies of their degradation and interaction with bone, Acta Biomater 2012 81230-8.Colloids Surf B Biointerfaces. 2009 Oct 1;73(1):1-9. doi: 10.1016/j.colsurfb.2009.04.030. Epub 2009 May 14.

[14] Derjaguin, B. (1939) A theory of interaction of particles in presence of electric double-layers and the stability of lyophobe colloids and disperse systems, Acta Phys. Chim., 10, 333-346.

[15] Derjaguin, B. and Landau, L. D. (1941) Theory of the stability of strongly charged lyophobic sols and of the adhesion of strongly charged particles in solutions of electrolytes Acta Phys. Chim., 14, 633-662.

[16] Bayoudh S., Othmane A, Mora L, Ben Ouada H., “Assessing bacterial adhesion using DLVO and XDLVO theories and the jet impingement technique", Colloids Surf B Biointerfaces. 2009 Oct 1;73(1):1-9. doi: 10.1016/j.colsurfb.2009.04.030. 